\title{
A Syllogism for Designing Collaborative Learning Technologies in the Age of AI and Multimodal Data
}

\author{
Mutlu Cukurova \\ UCL Knowledge Lab, University College London, \\ London, UK \\ m. cukurova@ucl.ac.uk
}

\begin{abstract}
Different paradigms of research interpret the social reality in different ways and these differences are not always apparent in technology enhanced learning research. However a paradigm's visibility and its elements' internal consistency are fundamental to the quality of research. As a philosophical position, a paradigm guides researchers to understand the nature of reality (ontology); how we create, acquire and disseminate knowledge (epistemology); and a systematic set of research strategy (methodology). In this research paper, the relationship between ontology, epistemology, and methodology is defined within the context of designing multimodal, AI technologies for collaborative learning. Two case study examples of inductive and deductive research methodologies are presented with the purpose of clarifying their differences in research outputs. Moreover, based on a recent literature review, it is presented that most empirical research in the field (40 out of 46) falls under the inductive methodology. Although, both deductive and inductive approaches are valuable for the advancement of the field; it is argued that the apparent lack of deductive investigations may lead researchers falling into technological determinism.
\end{abstract}

Keywords: Deductive Research · Inductive Research - Collaborative Learning Technologies · Artificial Intelligence

\section{Introduction}

Social reality is the main subject of educational research, but it is interpreted in multiple ways by different paradigms of research. In his seminal work "the structure of scientific revolutions", Thomas Kuhn conceptualises the term 'paradigm' in two different senses $(1962,1970)$. The first one defines a paradigm as "the entire constellation of beliefs, values, techniques, and so on, shared by the members of a given community" (p.175). The second one is as exemplary of past achievements, "the concrete puzzle-solutions which, employed as models or examples, can replace explicit rules as a basis for the solution of the remaining puzzles of normal science." (p.175). This paper refers to the first conceptualisation of 
paradigm. More specifically here, a paradigm is referred as a set of beliefs, values, and assumptions that are shared by the members of the research community that undertakes research into the design of collaborative learning technologies with AI techniques and multimodal data.

Unfortunately, often the paradigms of the research we are undertaking are not explicit to other researchers in the field, and in some cases, it is not even explicit to us. Optimistically, this is due to such assumptions and values being deeply embedded in our thinking, and even though we do not explicitly reflect upon them, our research actions are internally consistent within our paradigm. Pessimistically, we are constantly shifting between the ontology, epistemology, and methodologies of different paradigms, lacking an internally consistent way of thinking about the research we are undertaking. The purpose of this paper is to remind us that regardless of the technological development era we are operating in, it is not possible to undertake research without committing to a paradigm and their transparency and consistency is paramount to ensure research quality.

Every good researcher's decision to reject one paradigm almost simultaneously means that they make the decision to accept another, and ideally, the judgment leading to that decision involves an informed comparison of different paradigms with nature and with each other (Kuhn, 1970). However, whether it is conscious or not, in the research we undertake we take a decision to accept one paradigm which becomes apparent in our methodological decisions. These different methodologies we implement lead to different research products and outputs. This paper presents two recent research studies on the design of collaborative learning technologies within the context of multimodal learning analytics to exemplify the different research products created due to the use of different methodologies; even though both approaches have similar data sources, tools, research contexts, and purposes.

\section{The relationship between ontology, epistemology, and methodology}

As discussed in the introduction section, the social reality is interpreted by multiple perspectives and differences can best be understood by an analysis of the assumptions that underpin research. One set of assumptions are the ontological ones, which mainly aims to provide answers to the key question of how the reality is defined. Ontology asks philosophical questions such as, is social reality external to an individual - imposing itself from without - or is it the product of individual's consciousness? Ontology is the study of being and ontological assumptions are concerned with what constitutes reality. For example, if a researcher believes that the social reality is external to an individual, and it exists outside the existence of the individual inquiring about it, their ontological position can be considered as a realist, assuming the facts are independent of mind. Alternatively, if a researcher considers that the reality is the product of individual consciousness and its pure existence is dependent upon the individual who enquiries about it, they can be considered as an 'anti-realist'. For instance, in 
a constructivist paradigm it is argued that meaning does not exist in its own right; rather it is constructed by human beings as they interact and engage in interpretation. Constructivist ontological assumption is that the reality is socially constructed. The reality is perceived based on the very context in a given situation and can hardly be generalised into one common reality. This perception directly challenges the typical positivist view that reality perceived in one context could be transferred to another with a similar setting.

Table 1. Assumptions of different research paradigms of positivism and post-positivism about key concepts of research (Adapted from O'Leary (2004, p.7)

\begin{tabular}{|l|l|l|}
\hline Positivist Assumptions & Assumptions about & Post-positivist assumptions \\
\hline Knowable & $\Leftarrow$ the world $\Rightarrow$ & Ambiguous \\
Predictable & $\Leftarrow$ the world $\Rightarrow$ & Variable \\
Single truth & $\Leftarrow$ the world $\Rightarrow$ & Multiple reality \\
Empirical & $\Leftarrow$ the nature of research $\Rightarrow$ & Intuitive \\
Reductionist & $\Leftarrow$ the nature of research $\Rightarrow$ & Holistic \\
Objective & $\Leftarrow$ the researcher $\Rightarrow$ & Subjective \\
Removed expert & $\Leftarrow$ the researcher $\Rightarrow$ & Participatory \& Collaborative \\
Deductive & $\Leftarrow$ methodology $\Rightarrow$ & Inductive \\
Hypothesis-driven & $\Leftarrow$ methodology $\Rightarrow$ & Exploratory \\
Reliable & $\Leftarrow$ methodology $\Rightarrow$ & Dependable \\
Reproducible & $\Leftarrow$ methodology $\Rightarrow$ & Auditable \\
Often quantitative & $\Leftarrow$ findings $\Rightarrow$ & Often qualitative \\
Statistically significant & $\Leftarrow$ findings $\Rightarrow$ & Valuable \\
Generalisable & $\Leftarrow$ findings $\Rightarrow$ & Idiographic or transferable \\
\hline
\end{tabular}

Another set of assumptions outlined are epistemological. These assumptions relate to the questions about the nature of knowledge, how is knowledge created, what are its forms, and, how can it be communicated. In constructivism epistemological assumptions indicate that knowledge is subjective because it is socially constructed. Therefore, knowledge generation is bound to the context where the acquisition of knowledge is happening. It is argued that therefore the epistemological positioning of the constructivist paradigm seeks to understand how social actors recognise, produce, and reproduce social actions and how they come to share an intersubjective understanding of specific life circumstances. On the other hand, post-positivists believe that knowledge about reality could be gained through an empirical evaluation. Nevertheless, because of the researcher limitations, any theories and knowledge well-established are only tentatively proven until disapproved by new evidence and findings (Mertens, 2014).

Ontological and epistemological positions play a direct role determining the research methodology that will be implemented in research. A methodology is the entire set of research strategies. In other words, it is the summary of the research process that ensures the data collected and the chosen study context are in line with the knowledge that research question(s) intend to obtain. And finally, as a component of the methodology, methods are data collection and analysis 
techniques. For instance, a researcher taking a positivist paradigm tend to form an abstraction of reality through primarily quantitative models using an experimental or quasi-experimental design with deductive hypotheses (Mertler, 2016). They will be taking an "outsider" position aiming to test their hypotheses. In contrast a constructivist position researcher will often use more flexible research methodologies in which the subjectivities are clearly defined and explained. They may form part of the research, taking an "insider" position, acknowledging that the knowledge investigated is socially constructed and inductively investigated.

\section{Two Case Studies with Different Research Paradigms to Design Collaborative Learning Technologies}

Here, inductive and deductive research methodologies will be focussed to exemplify two different paradigms' research approaches. The differences in the research products will be illustrated with two case studies both aiming to design collaborative learning technologies with multimodal data collected from the same project-based learning context.

\subsection{Case study 1: Deductive Approach}

The case study example for the deductive approach is a recent paper by Cukurova et al., (2018). The purpose of the paper is to identify students' effective collaborative problem-solving (CPS) behaviours in real-world teaching environments so that technology that observes such behaviours can be designed and can be used to support skill development. The article starts with a definition of CPS and presents a literature review on the mechanisms through which CPS may influence cognition and support deeper learning. The researchers identified four key constructs from the learning sciences literature that are argued to be relevant to the process of CPS, namely synchrony, individual accountability, equality, and intra-individual variability and experimentally investigate their relation to CPS. Their results show that students in high competence CPS groups have member students who have high and equal scores for physical interactivity and low and equal scores for intra-individual variability. Moreover, high competence CPS groups appear to have high levels of student synchrony and individual accountability values. Based on these results, taking a deductive approach, the authors argue that the future research will involve attempts to design a piece of technology to automate this process of interpreting student behaviours using multimodal learning analytics in order to provide real-time feedback to students and teachers about learning processes. More recently, they designed a computer vision system based on multimodal data and deep neural networks that is able to detect those key constructs of CPS that are deductively created in NISPI framework (Landolfi et al., under review). 


\subsection{Case study 2: Inductive Approach}

With the same purpose of identifying and supporting students' effective CPS behaviours in project-based learning environments, Spikol et al., (2018) investigates the potential of data collected from high-fidelity synchronised multimodal recordings of small groups of learners interacting. As opposed to the NISPI paper's approach of deductively identifying key constructs of CPS, in this article the authors process and extract different aspects of the students' interactions to identify which features are representative of success in educational contexts of open-ended project work. Inductively exploring multiple data sources with different machine learning approaches, the authors investigate the potential of number of the faces looking at the screen, the mean distance between learners, the mean distance between hands, the mean hand movement speed, the mean audio level, project complexity, active hardware and software blocks, and the students' work phases. They conclude that the distance between learners' hands and faces is a strong predictor of student collaboration, whereas other features do not predict the project outcomes and student collaboration.

\section{Discussion and Conclusions}

Case studies presented above have the same goal of designing a piece of technology that would support collaborative learning in project-based learning environments, yet the decisions that are taken during the research process are distinct from each other. More importantly, even though both research studies investigate almost identical research contexts with almost identical data sources and almost identical data intelligence tools, due to different paradigms underpinning two case studies, they produce completely different research products. At this stage, one could argue for the potential superiority of one approach over the other. However, this is not the point of this paper. Ontology and epistemology are axiological, that means they are related to values (Carter, \& Little, 2007). Therefore their associated methodologies are bound to certain assumptions and values. The purpose here is not to argue for, or against, any of them as they are incommensurable. However, it is to argue that good quality research should clearly explain the methodological decisions taken and argue for the internal consistency of its elements (Mantzoukas, 2004). As long as such internal consistency is provided, arguments that relate to ultimate superiority of a certain paradigm over other would require repression of existing useful logics (Carter, \& Little, 2007), and the world of ideas does not call for one true 'logic' (Kaplan, 1964).

Although, there are research papers emphasising on the value of considering epistemic beliefs in the design of learning analytics (c.f. Knight, Shum, \& Littleton, 2014); epistemic transparency is often not present. For instance, in Worsley (2018)'s recent review of the field of multimodal learning analytics (MMLA), eighty-two papers (forty-six empirical) were identified and there is hardly any mention of the operated research paradigms in these identified papers. Considering the emerging nature of the field, perhaps, this is expected. However, based 
on considerations exemplified in two case studies above, it also becomes apparent that most research published in the field takes an inductive approach (fourty out of fourty-six empirical studies). This might be problematic; not due to the inferiority of this approach over the other, but due to the potential monopoly of one particular research paradigm in the field. More varied approaches, such as the deductive approach presented in case study one, can lead to MMLA's richer contribution to Educational contexts. For instance, the prevalence of inductive approach might lead to prioritising the existing data sources and tools over designing new ones that are based on the requirements of existing research in learning sciences and may lead to the production of research outcomes that are not of great value to authentically situated learning environments. This limits the ways we can challenge the technology, and might ultimately lead to technological determinism.

\section{References}

1. Carter, S. M., \& Little, M. (2007). Justifying knowledge, justifying method, taking action: Epistemologies, methodologies, and methods in qualitative research. Qualitative health research, 17(10), 1316-1328.

2. Cukurova, M., Luckin, R., Milln, E., \& Mavrikis, M. (2018). The NISPI framework: Analysing collaborative problem-solving from students' physical interactions. Computers \& Education, 116, 93-109.

3. Kaplan, A. (1964). The conduct of inquiry: Methodology for behavioral science. San Francisco: Chandler.

4. Knight, S., Shum, S. B., \& Littleton, K. (2014). Epistemology, assessment, pedagogy: where learning meets analytics in the middle space. Journal of Learning Analytics, 1(2), 23-47.

5. Kuhn, Thomas S. (1962) The Structure of Scientific Revolutions. Chicago: University of Chicago Press.

6. Landolfi, L., Ruffaldi, E., Cukurova, M., Spikol, D. (under review).Collaboration analysis of students' physical interaction based on neural networks and body pose. IEEE Transactions on Learning Technologies.

7. Mantzoukas, S. (2004). Issues of representation within qualitative inquiry. Qualitative Health Research, 14(7), 994-1007.

8. Mertens, D.M., (2014). Research and evaluation in education and psychology: integrating diversity with quantitative, qualitative, and mixed methods. Sage.

9. Mertler, C. A. (2016). Action research: Improving schools and empowering educators. Sage Publications.

10. Spikol D, Ruffaldi E, Dabisias G, Cukurova M. (2018) Supervised machine learning in multimodal learning analytics for estimating success in project-based learning. Journal of Computer Assisted Learning, doi.org/10.1111/jcal.12263.

11. O'leary, Z. (2004). The essential guide to doing research. London: Sage. 12. Worsley, M. (2018). Multimodal Learning Analytics Past, Present, and, Potential Futures. Companion Proceedings of LAK18, CrossMMLA, 2018. 